\title{
The Era of Nerve Transfers in Tetraplegic Patients: Our Multidisciplinary Experience
}

\author{
Morini $\mathrm{S}^{1 *}$, Colzani $\mathrm{G}^{2}$, Lavorato $\mathrm{A}^{3}$, Vincitorio $\mathrm{F}^{3}$, Titolo $\mathrm{P}^{2}$, Garbossa $\mathrm{D}^{3}$, Battiston $\mathrm{B}^{2}$, Isoardo $\mathrm{G}^{4}$, \\ Dimanico $U^{5}$, Negri $S^{5}$, Petrozzino $S^{5}$, Minetto $M^{1}{ }^{1}$ and Massazza $G^{1}$ \\ ${ }^{1}$ Division of Physical Medicine and Rehabilitation, Department of Surgical Sciences, University of Turin, Italy \\ ${ }^{2}$ OU Traumatology-Reconstructive Microsurgery, Department of Orthopaedics and Traumatology, CTO Hospital, Italy \\ ${ }^{3}$ OU Neurosurgery, Department of Neurosciences, University of Turin, Italy \\ ${ }^{4}$ Centre for Pain Treatment, Department of Anesthesiology, Resuscitation and Intensive Care, Città della Salute e della Scienza di Torino \\ - Molinette Hospital, Italy \\ ${ }^{5}$ Department of Physical Medicine and Rehabilitation, Unipolar Spinal Unit, AOU Città della Salute e della Scienza di Torino, Italy \\ *Corresponding author: Silvia Morini, Division of Physical Medicine and Rehabilitation, Department of Surgical Sciences, University \\ of Turin, Via Gianfranco Zuretti, 24, Turin, Italy
}

ARTICLE INFO

Received: 幽 June 16, 2021

Published: 蔧 June 25, 2021

Citation: Morini S, Colzani G, Lavorato A, Vincitorio F, Titolo P, et al., The Era of Nerve Transfers in Tetraplegic Patients: Our Multidisciplinary Experience. Biomed J Sci \& Tech Res 36(5)-2021. BJSTR. MS.ID.005908.

Keywords: Nerve Transfers; Tetraplegia; Multidisciplinary Approach; Pre-Operative Evaluation; Upper Limb Rehabilitation; Hand Rehabilitation

Abbreviations: SCI: Spinal Cord Injuries; ADL: Activities of Daily Life; EMG: Electromyography; NCS: Nerve Conduction Study; ROM: Range of Motion

\section{ABSTRACT}

Objective: The aim of our work is to propose a systematic approach in the management of the upper limb rehabilitation in tetraplegic patients, focused on the preoperative rehabilitation aspects that must be tailored to the specific therapeutic path, in order to assure the best conditions both before the intervention or the conservative management, and optimize the results.

Methods: Evaluation criteria, surgical and rehabilitation timing with reference to the recent literature are reported. Timing and objectives of upper limb rehabilitation in tetraplegic patients are discussed, focusing on obstacles in the management of patients that can be overcome with a multidisciplinary approach.

Results and Discussion: The upper limb diagnostic-therapeutic path of tetraplegic patients is developed point by point, starting from the evaluation, the indications, therapeutic options, surgical timing and focusing on the role of pre-operative rehabilitation. At present, there is consensus regarding the need for physiotherapy aimed to maintain flexible joints if surgery is planned or learning compensatory mechanisms for candidates to a conservative management. The application timing of rehabilitation protocols in relation to the different surgical strategies is of great importance, as the choice to perform the classic tendon transfers or the most innovative nerve transfers influences the entire therapeutic path.

Conclusion: The management of the tetraplegic patient requires a coordinated and multidisciplinary approach, which can be intended to implement residual functions, or prepare for surgery. Preoperative physiotherapy must take into account both the best personalized protocol and the timing dictated by the type of surgical choice.

\section{Introduction}

The annual incidence of Spinal Cord Injuries (SCI) is estimated around 40 to 100 cases per million worldwide, and mainly affects young adults involved in sports or road accidents [1-3]. The cervical tract is compromised in about half of the cases and could lead to the development of different degrees of tetraplegia [4]. The motor deficit can affect the four limbs and the trunk in different ways, but the constant outcome in such situations is the almost complete 
compromise of the patient's autonomy, which entails the need of a continuous and lifelong support from the caregivers for the activities of daily life (ADL) such as eating, dressing and providing personal hygiene [3]. Despite SCI inevitably led to a significative shortening of life expectancy for a very long time, currently these patients show an improvement of their perspectives thanks to the advances in medical care [5]. In this context, the role of surgery and rehabilitation in acute and chronic phase is fundamental. The goal is therefore no longer just to keep these individuals alive, but to provide them with effective means to improve their quality of life [5]. Restoring a certain functionality of the upper limbs - like wrist extension and handgrip [6,7] appears then of significant importance in this perspective [8,9].

\section{Materials and Methods}

As a team, we have developed over years a multidisciplinary approach to the tetraplegic patient. In this article we report our evaluation criteria and our methods of approach, discussing them point by point in the light of recent literature. Our evaluation timing is reported as well, considering the potential surgical treatment and the objectives of upper limb rehabilitation in this type of patients. In the final part of this paper, the obstacles encountered in the management of the patient's diagnostic-therapeutic path are discussed, focusing on the aspects that can be overcome with a multidisciplinary approach.

\section{Results and Discussion}

\section{Clinical Examination and Diagnostics}

The clinical examination and instrumental diagnostics play an important role from the very beginning to establish the residual functions and choose the possibles surgical options.

\section{Muscle Testing}

The patient is inlaid from a clinical and functional point of view in one of the groups defined in the classification of Allieu in Giens, which takes into account the residual active muscles [10]. The evaluation of muscle strength of potential redundant donors and putative recipients is carried out according to the British Medical Research Council scale (MRC) [10]. The assessment of the patient must be carried out bilaterally to allow the understanding of the global use pattern, the dominant side, the identification of the major critical issues, the overall residual use of the limbs resulting from the progressive adaptation to his condition of disability. Providing truncal support allows for a more focused and realistic examination of the muscular function of the upper extremities. In the surgical choices it is also necessary to consider the possibility of exploiting not only muscle motors but also other strategies such as the force of gravity and the tenodesis effect, in particular as regards the functionality of the fingers of the hand. It is important to consider the possible presence of spasticity that may limit the use of some muscle groups as potential donors. A donor muscle must be healthy, of adequate strength (al least M4), and it must not be compromised by scars nor have already been reinnervated. Ideally, in tendon transfers, the donor should be similar to the recipient in structure, be synergistic and have an adequate soft tissue environment along the transfer route [11-13]. A similar discourse concerns nerve donors which, however, extend the surgical possibilities also to different districts and to the sensitive component.

\section{Sensitivity Testing}

For the sensitive evaluation the two-point discrimination test in currently used $[10,14]$. In cases of severe sensory deficits, the patient's ocular control should also be evaluated because it can play an important role in motor control after surgery. Identifying the areas of residual sensitivity and quantifying their extent and localization certainly helps the understanding of the level of injury. It is clear however that the restoration of motility is in any case a priority in the operative strategy with respect to the recovery of sensitivity, and the surgical gestures in this sense are always a useful but not strictly indispensable corollary.

\section{Joint Mobility}

The recovery of a motor function necessarily presupposes the absence of joint stiffness in that district. It is advisable to perform radiographs or CT scans in order to study the osteoarticular component and therefore evaluate the need for a preliminary surgical step as arthrolysis, removal of any post-traumatic ossifications or possible corrective osteotomies. Otherwise, any further reconstructive intervention risks to be unsuccessful.

\section{Electrodiagnostic Testing}

In the preoperative diagnostic phase, we can make use of instrumental evaluations such as the electromyography (EMG) and the nerve conduction study (NCS). The first exam allows to know how muscles respond to nerve signals and can be performed at rest or during voluntary recruitment. The second one measures how the electrical signal runs along the nerve. In most cases, both these evaluations are performed at the same time. Usually they are performed on the occasion of the initial clinical assessment which is normally carried out a few months after trauma, and are not routinely repeated unless clinical changes arise. The crucial point is that they are performed by an experienced electrophysiologist because an incorrect, incomplete and approximate evaluation can negatively affect the subsequent therapeutic choices.

\section{Indication for Surgery}

Surgical indications depend on the level of injury and the type of deficit resulting from it. We often observe conditions of partial and asymmetrical paralysis, so a careful and reiterate evaluation is essential. To undergo surgery the patient must result stabilized from a general point of view (including the psychological aspect) 
and should not have shown a significant functional recovery after the first six months after trauma [15]. Any recent improvement in motor or sensory function have to be investigated and followed, observing the patient closely until the spontaneous recovery shows a stop, in order to maximize the potentiality of the natural course before considering any surgical options. The possible presence of spasticity must be investigated and treated before elaborating the surgical strategy [16]. Joint contractures are contraindications for surgery because they generally lead to poor outcomes. If stiffness is evident, it must first be corrected if possible, in order to gain an adequate passive range of motion.

To decrease surgical risks, the patient should be clinically stable, without active infections or relevant systemic diseases. Chronic pain must be sufficiently controlled. Moreover, they are fragile persons, with a precarious psychological balance. The social and familial support is particularly important [17]. Both patients and caregivers must demonstrate that they are mentally prepared for surgery. They should have realistic expectations on functional outcomes and recovery times. They have to be motivated to strictly follow the pre- and post-operative rehabilitation program and they must have adequate psychological support in order not to reduce their compliance and compromise the results.

\section{Surgical Options}

To restore lost functions, tendon transfers are a safe and reliable option, and have been the mainstay of functional upper limb surgery in tetraplegia for decades [18-20]. Their purpose is to restore active motor control over some essential functions of an anatomical segment, by transposing one or more healthy muscles whose function can be sacrificed without significant residual damage [21]. These techniques can also be performed many years after the trauma, as long as the joints are passively mobile, and the donor muscles have adequate strength (at least M4 according to the British Medical Research Council scale) [20]. In more recent times, the increasing use of nerve transfer techniques has revolutionized and implemented the possibilities of functional recovery in tetraplegic patients, expanding the opportunities even in those cases where it was not possible to obtain any improvement with tendon transfers alone [22]. Nerve transfer strategies adopted in tetraplegia are inspired by those already consolidated in the treatment of patients suffering from brachial plexus paralysis.

The goal is to exploit the axonal regeneration of healthy expendable nerves that arise above the lesion level, transferred to nerves that are no longer functioning being placed below it $[23,24]$. Among the advantages of these techniques, we find the possibility of obtaining a more natural and safe recovery of strength [25] as the position and the excursion of the muscles are preserved $[23,24]$. It is also possible to act on the restoration of sensitivity. These techniques also allow for more versatile strategies, such as the possibility to select some functional priorities, for example the choice to reinnervate the more distal districts to allow the recovery of a certain degree of pinch and grip strength [26,27]. The use of nerve transfers can also be studied in combination with some tendon transfers, as an accurate choice of nerve donors does not necessarily jeopardize the subsequent use of other muscles for traditional palliative surgery [22]. In cases where it is not possible to use neither nerve nor tendon transfers, techniques such as tenodesis and arthrodesis remain feasible to guarantee some limited functions.

\section{Timing for Surgery}

Appropriate surgical timing is an extremely important aspect to optimize functional results. To proceed with traditional tendon transfers it was historically advisable to wait at least one year after the trauma, assuming that for that time any possible spontaneous neurological recovery had been achieved $[21,28]$. The situation differs for nerve transfers, as it is recommended to perform them within one year of the injury before muscle denervation becomes too severe $[29,30]$. However, the precise timing is still debated, as it can be influenced by multiple factors, mainly related to the state of innervation of the muscle based on the level of injury. About that, three different categories of muscles can be identified in patients with $\mathrm{SCI}$, and this aspect must be taken into consideration because it influences the surgical timing and the chances of recovery [23,31]. The first group includes muscles innervated by the supralesional medullary segment: these are functional, under volitional cortical control, and their nerves can be used as donors for transfers.

The second group comprises muscles pertaining to the infralesional segment. They are disconnected from the descending motor tract, but anatomically connected to their correspondent sub-lesional spinal cord anterior horn cells via intact reflex arcs, resulting in a pure upper motor neuron disfunction. These muscles are functionally paralyzed but maintain the ability to successful distal reinnervation. If electrically stimulated, they show contractions. Surgery in this case appears relatively timeindependent: a nerve transfer can switch motor control from a redundant and expendable donor to a muscle group distal to the lesion site bypassing the level of injury [32,33]. In this way, the functional motor units are reconnected to the central nervous system, potentially restoring volitional control. Due to the slow neuromuscular degeneration, nerve transfers in these cases can be ideally performed at any time after SCI. Bertelli et al. reported transfers carried out 18 months after trauma showing functional muscle reinnervation [34].

The third group includes muscles innervated by neurons at the injury site. The extent of the segment can vary a lot, being almost negligible in an ideally clear-cut SCI or with massive involvement including the anterior horn cells and cervical roots. These cases are 
similar to peripheral nerve injuries, resulting in a prevailing lower motor neuron dysfunction with motor endplate changes and typical denervation pattern. The correct timing for surgery significantly influences the outcomes in these cases [35]. Nerve donors should be used to restore voluntary control reinnervating muscles that would otherwise undergo irreversible atrophy. The neuromuscular degeneration takes place gradually over the course of one year, so nerve transfer surgery should be ideally performed within 6-12 months after injury [36].

\section{The Role of Pre-Operative Rehabilitation}

Based on the decisions agreed in the initial multi-disciplinary assessment, rehabilitation can take on different meanings and objectives. It can therefore assume a preparatory role for surgery or guide in the acquisition of compensatory skills if the realistic possibilities for improvement related to the intervention are minimal or it is completely excluded. Where it is feasible, surgery can truly guarantee a noticeable functional improvement for the person with SCI. These techniques, particulary in nerve transfers, are constantly growing, always providing new possibilities for recovery, $[37,38]$ and patients must be made aware of the avaiable reconstructive options [7]. For example, regaining grip strength eliminates or reduces the need for adaptive equipment. The patient can then return to groom, feed, catheterize by himself, and this gives him an important component of autonomy. The ability to manipulate objects, write, perform productive works plays a decisive role in regaining spontaneity and self-confidence, thus helping the patient also from a psychological point of view $[12,20]$.

In these cases, pre-operative rehabilitation should be addressed toward prevention of contractures and maintenance of a soft, supple hand with preserved range of motion (ROM). The hand therapist thus has to avoid claw hand posturing, interphalangeal contractures and thumb adduction contractures [39]. All the efforts should be aimed at the preparation for surgery above all any compensatory strategies. If the patient is not eligible for surgery or thinks he cannot manage the proposed surgical-rehabilitation path, a conservative approach is always feasible. In these cases the rehabilitation involves the search for functional compensation. For some patients, one of the main common compensatory strategies is obtaining an active or passive tenodesis grip. To achieve that, the most common approach is to splint the fingers and wrist, in order to create a progressive shortening of the flexors muscles, such as to allow a rudimentary grasping ability [40].

\section{The Importance of a Multidisciplinary Approach: A Patient-Tailored Treatment}

During the initial multidisciplinary assessment, the interaction between all the medical team members and the patient with his caregivers is crucial. It is on these occasions that an adequate communication and explanation on the real therapeutic possibilities must take place in order to personalize the treatment path. Different strategies are often feasible, based on the requests, expectations and compliance of the patient. What is important to convey is the concept that surgery and rehabilitation are always strictly interdependent, and the choice of a type of path, conditions all the phases from the very beginning. Generally, a conservative approach is still widely shared, even if it is often the result of incomplete trust or knowledge of surgical possibilities. The school of thought behind this strategy is to promote flexor muscle retraction through splinting starting from the early stages of rehabilitation, in order to obtain a tenodesis grip. The risk is these cases is to undertake a compensatory re-education process without first having carried out an adequate multidisciplinary assessment with an expert surgical team, and which therefore may preclude the planning of a time-dependent functional surgery.

From a more modern perspective, it is important not to neglect this aspect of multidisciplinary discussion and sharing, taking always into account that if the patient is eligible for surgery, the best rehabilitative approach is to preserve the natural muscular length and joint mobility. In fact, contractures resulting from the intentional development of a stiff hand with minimal grip function, as it happens in most conservative protocols, are then difficult or impossible to treat in a reasonable amount of time in the event that a functional surgery is subsequently opted and represent a relative contraindication because they can compromise the final results leading to poor outcomes $[37,38,41]$. The emergent model of management of such situations provides that the patient and his caregivers are involved in the decision-making process from the very beginning, and that they are aware that certain options may exclude other therapeutic alternatives. All the proper information about surgical and conservative treatments must be given, always considering the most up-to-date strategies but not forgetting the most consolidated ones. It is crucial to clarify the reasonable motor recovery times and the real possibilities for improving in daily life, not neglecting the need for constant commitment in the pre- and post-operative rehabilitation phase [42].

This is even more important if nerve surgery is planned, as the times are longer than the traditional tendon transfers. Despite the continuous evolution of functional surgery techniques has shown the possibility to obtain results unthinkable until a few decades ago, it is still an underused and quite unknown field, often determining a gap between what is possible and what is applied $[5,26]$. The reasons behind this paradox are to be found in the persistence of inadequate information and interaction between professionals, which therefore leads to the lack of an efficient reference network that can deal with these patients in adequate times [7]. Since functional surgery is carried out only in few referral hospitals, having an effective communication with professionals on the territory is crucial, in order to address the patients in the 
correct times and conditions that allow the maximum surgical and rehabilitative possibilities [43]. Once the most appropriate therapeutic strategy has been set, a continuous interaction between the surgeons and the rehabilitation team and caregivers is essential for the optimization of the results.

\section{Conclusion}

The patient with cervico-medullary trauma requires an integrated and multidisciplinary approach in all phases of care. In this scenario, the preoperative evaluation assumes fundamental importance. Both the patient and the caregivers must be adequately informed about the possible surgical options, the related risks, the real desirable advantages, the recovery times, and the subsequent re-education phases. The contemporary presence of the competent surgeons, the physiatrist, the physiotherapist, the occupational therapist plays a central and indispensable role in this context. The operative strategy must be tailored on the patient's specific needs. First, he must be asked which functions he would like to implement and must be informed about the real feasibility in achieving them. Then, the choice of the donor nerves or tendons must be subordinated to a careful clinical and electrophysiological preliminary evaluation. Keeping in mind the evolution timing of the post-traumatic neurological state, the ideal time to perform this preliminary assessment is at about 4 months after injury. Then, a second visit is scheduled at about 6 months: if there is no evidence of functional recovery and the examination is comparable to the previous one, it is appropriate to proceed with the surgical planning. In the elapsing period it is very important to refer the patient to an appropriate rehabilitation program that in these cases could act as a preparation for the subsequent surgery.

\section{Conflict of Interest}

The authors declare that they have no conflict of interest.

\section{References}

1. James SL, Bannick MS, Montjoy Venning WC, Lucchesi LR, Dandona L, et al. (2019) Global, regional, and national burden of traumatic brain injury and spinal cord injury, 1990-2016: A systematic analysis for the Global Burden of Disease Study 2016. The Lancet Neurology 18(1): 56-87.

2. Singh A, Tetreault L, Kalsi S, Aria R, Michael N, et al. (2014) Clinical Epidemiology Dovepress Global prevalence and incidence of traumatic spinal cord injury. Clinical Epidemiology.

3. (2016) WHO. International perspectives on spinal cord injury. WHO.

4. Ackery A, Tator C, Krassioukov A (2004) A global perspective on spinal cord injury epidemiology. Journal of Neurotrauma 21(10): 1355-1370.

5. Zlotolow DA, Lipa B, Pahys JM (2019) Team Approach: Treatment and Rehabilitation of Patients with Spinal Cord Injury Resulting in Tetraplegia. JBJS Reviews 7: 1-11.

6. Snoek GJ, Ijzerman MJ, Hermens HJ, Maxwell D, Biering Sorensen F (2004) Survey of the needs of patients with spinal cord injury: Impact and priority for improvement in hand function in tetraplegics. Spinal Cord 42: 526-532.

7. Curtin CM, Gater DR, Chung KC (2005) Upper extremity reconstruction in the tetraplegic population, a national epidemiologic study. Journal of Hand Surgery 30: 94-99.
8. Anderson KD (2004) Targeting recovery: Priorities of the spinal cordinjured population. Journal of Neurotrauma 21(10): 1371-1383.

9. Snoek GJ, Ijzerman MJ, Hermens HJ, Maxwell D, Biering-Sorensen F (2004) Survey of the needs of patients with spinal cord injury: Impact and priority for improvement in hand function in tetraplegics. Spinal Cord 42(9) : 526-32.

10. Moberg E, L McDowell C, H House J (1989) Third International conference on Surgical Rehabilitation of the upper limb in tetraplegia (quadriplegia). Journal of Hand Surgery 14(6): 1064-1066.

11. Fridén J, Gohritz A, Turcsányi I, Ejeskär A (2012) Restoration of active palmar abduction of the thumb in tetraplegia by tendon transfer of the extensor digiti minimi to abductor pollicis brevis. The Journal of Hand Surgery, European 37(7): 665-72.

12. Hentz V, Ladd A (2002) Long-Term Outcomes in Surgical Rehabilitation of the Upper Limb in Tetraplegia. Orthopedics.

13. Fridén J, Gohritz A (2012) Novel Concepts Integrated in Neuromuscular Assessments for Surgical Restoration of Arm and Hand Function in Tetraplegia. Physical Medicine and Rehabilitation Clinics of North America 23(1): 33-50.

14. McDowell C, Moberg E (1986) Surgery JH-J of H, undefined. The second international conference on surgical rehabilitation of the upper limb in tetraplegia (quadriplegia).

15. Davidge KM, Fox IK (2008) Nerve Transfer Procedures for Tetraplegia n.d 24(2): 157-60.

16. Fridén J, Reinholdt C (2008) Current concepts in reconstruction of hand function in tetraplegia. Scandinavian Journal of Surgery 97(4): 341-346.

17. Lee SK, Wolfe SW (2012) Nerve Transfers for the Upper Extremity. Journal of the American Academy of Orthopaedic Surgeons 20(8): 506517.

18. Bednar MS (2016) Tendon Transfers for Tetraplegia. Hand Clinics 32(3): 389-396.

19. Dunn JA, Sinnott KA, Rothwell AG, Mohammed KD, Simcock JW (2016) Tendon transfer surgery for people with tetraplegia: An overview. Archives of Physical Medicine and Rehabilitation 97(6): S75-80.

20. Fridén J, Gohritz A (2015) Tetraplegia management update. Journal of Hand Surgery 40(12): 2489-500.

21. Heest AE van (2010) Green's Operative Hand Surgery. pp. 1209-34.

22. Titolo P, Fusini F, Arrigoni C, Isoardo G, Conforti L, et al. (2019) Combining nerve and tendon transfers in tetraplegia: a proposal of a new surgical strategy based on literature review. European Journal of Orthopaedic Surgery and Traumatology 29(3): 521-530.

23. Senjaya F, Midha R (2013) Nerve Transfer Strategies for Spinal Cord Injury. World Neurosurgery 80(6): e319-e326.

24. Ray WZ, Chang J, Hawasli A, Wilson TJ, Yang L (2015) Motor nerve transfers: A comprehensive review. Neurosurgery 78(1): 1-25.

25. Cain SA, Gohritz A, Fridén J, Van Zyl N (2015) Review of upper extremity nerve transfer in cervical spinal cord injury. Journal of Brachial Plexus and Peripheral Nerve Injury 10: e34-42.

26.Zlotolow DA (2011) The role of the upper extremity surgeon in the management of tetraplegia. Journal of Hand Surgery 36(5): 929-935.

27. Limthongthang R, Bachoura A, Songcharoen P, Osterman AL (2013) Adult brachial plexus injury. Evaluation and management. Orthopedic Clinics of North America 44(4): 591-603.

28. Kakulas BA (2004) Neuropathology: The foundation for new treatments in spinal cord injury. Spinal Cord 42(10): 549-563.

29. Bertelli JA, Ghizoni MF, Tacca CP (2011) Transfer of the teres minor motor branch for triceps reinnervation in tetraplegia: Case report. Journal of Neurosurgery 114(5): 1457-60. 
30. Waters RL, Sie IH, Gellman H, Tognella M (1996) Functional hand surgery following tetraplegia. Archives of Physical Medicine and Rehabilitation 77(1): 86-94.

31. Fox IK (2016) Nerve Transfers in Tetraplegia. Hand Clinics 32(2): 227 42 .

32. Oppenheim JS, Spitzer DE, Winfree CJ (2009) Spinal cord bypass surgery using peripheral nerve transfers: Review of translational studies and a case report on its use following complete spinal cord injury in a human: Experimental article. Neurosurgical Focus 26(2): 1-6.

33. Coulet B, Allieu Y, Chammas M (2002) Injured metamere and functional surgery of the tetraplegic upper limb. Hand Clinics 18(3): 399-412.

34. Bertelli JA, Ghizoni MF (2013) Single-stage surgery combining nerve and tendon transfers for bilateral upper limb reconstruction in a tetraplegic patient: Case report. Journal of Hand Surgery 38(7): 1366-1369.

35. Vincitorio F, Cofano F, Colzani G, Titolo P, Lavorato A, et al. (2020) Letter to the Editor Regarding "Opinion Piece: Microsurgery in COVID-19Positive Patients." World Neurosurgery 139: 712-713.

36. Khalifeh JM, Dibble CF, Van Voorhis A, Doering M, Boyer MI, et al. (2019) Nerve transfers in the upper extremity following cervical spinal cord injury. Part 2: Preliminary results of a prospective clinical trial. Journal of Neurosurgery: Spine 31: 641-53.

37. Bertelli JA, Ghizoni MF (2015) Nerve transfers for elbow and finger extension reconstruction in midcervical spinal cord injuries. Journal of Neurosurgery 122(1): 121-127.

\section{ISSN: 2574-1241}

DOI: $10.26717 /$ BJSTR.2021.36.005909

Patrick A Grant. Biomed J Sci \& Tech Res

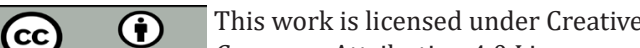
Commons Attribution 4.0 License

Submission Link: https://biomedres.us/submit-manuscript.php
38. Bertelli JA, Tacca CP, Ghizoni MF, Kechele PR, Santos MA (2010) Transfer of Supinator Motor Branches to the Posterior Interosseous Nerve to Reconstruct Thumb and Finger Extension in Tetraplegia: Case Report. The Journal of Hand Surgery 35(10): 1647-1651.

39. Keith MW, Lacey SH (1991) Surgical Rehabilitation of the Tetraplegic Upper Extremity. Neurorehabilitation and Neural Repair 5: 75-87.

40. Krajnik SR, Bridle MJ (1992) Hand splinting in quadriplegia: current practice. The American Journal of Occupational Therapy: Official Publication of the American Occupational Therapy Association 46(2): 149-56.

41. Khalifeh JM, Dibble CF, Van Voorhis A, Doering M, Boyer MI, et al (2019) Nerve transfers in the upper extremity following cervical spinal cord injury. Part 1: Systematic review of the literature. Journal of Neurosurgery: Spine 12: 1-12.

42. Baldassarre BM, Vincitorio F, Lavorato A, Colzani G, Titolo P, et al. (2020) Principles of cortical plasticity in peripheral nerve surgery. Surgical Technology International 36: 1-9.

43. Fattal C, Coulet B, Verollet C, Rouays Mabit H, Schindler F, et al. (2008) La chirurgie fonctionnelle du ou des membres supérieurs chez la personne tétraplégique. Lettre de Medecine Physique et de Readaptation 24: 6772.

$\begin{array}{ll}\text { BIOMEDICAL } & \text { Assets of Publishing with us } \\ \text { RESEARCHES } & \text { - Global archiving of articles } \\ \text { - Immediate, unrestricted online access }\end{array}$

